\title{
Calorimetry of Nucleic Acids
}

A number of units in this chapter describe a collection of techniques for evaluating the structures of nucleic acids. These techniques have been employed to generate a substantial and rapidly expanding database of nucleic acid structures. Yet it has become apparent that thermodynamic as well as structural information is required to develop a clear understanding of the complex relationships between structure, energetics, and biological function. In recognition of this requirement, the number of studies designed to characterize the thermodynamics of a broad range of nucleic acid structures has increased dramatically in recent years. This unit describes the application of calorimetry to characterize the thermodynamics of nucleic acids, specifically, the two major calorimetric methodologies that are currently employed: differential scanning and isothermal titration calorimetry.

Differential scanning calorimetry (DSC; Basic Protocol 1) is used to study thermally induced order-disorder transitions in nucleic acids. A DSC instrument measures, as a function of temperature $(T)$, the excess heat capacity $\left(C_{\mathrm{p}}^{\mathrm{ex}}\right)$ of a nucleic acid solution relative to the same amount of buffer solution. A single DSC profile provides a wealth of both thermodynamic and extrathermodynamic information, much of which cannot be obtained by any other technique. Specifically, from a single curve of $C_{\mathrm{p}}^{\mathrm{ex}}$ versus $T$, one can derive the following information (see Commentary): the transition enthalpy $(\Delta H)$, entropy $(\Delta S)$, free energy $(\Delta G)$, and heat capacity $\left(\Delta C_{\mathrm{p}}\right)$; the state of the transition (two-state versus multistate); and the average size of the molecule that melts as a single thermodynamic entity (i.e., the size of the cooperative unit).

Isothermal titration calorimetry (ITC; Basic Protocol 2) is used to study the hybridization of nucleic acid molecules at constant temperature. In a typical ITC experiment, small aliquots of a titrant nucleic acid solution are added to an analyte nucleic acid solution (with the analyte nucleic acid being complementary to the titrant nucleic acid) and the released heat is monitored. Judicious selection of nucleic acid concentrations and buffer conditions results in a titration curve that can be analyzed to yield the stoichiometry of the association reaction $(n)$, the enthalpy of association $(\Delta H)$, the equilibrium association constant $(K)$, and thus the free energy of association $(\Delta G)$. Once $\Delta H$ and $\Delta G$ are known, the entropy of association $(\Delta S)$ can also be derived. Thus, as noted above for DSC, a single ITC experiment also yields a wealth of thermodynamic information about the association reaction. Repetition of the ITC experiment at a number of different temperatures yields the $\Delta C_{\mathrm{p}}$ for the association reaction from the temperature dependence of $\Delta H$.

\section{STRATEGIC PLANNING}

\section{General Considerations}

The accuracy of the thermodynamic data derived from the calorimetric protocols described in this unit depends to a great extent on the purity of the nucleic acids being studied and the accuracy with which the concentrations of the experimental nucleic acid solutions have been determined. All nucleic acids should be devoid of protein contaminants, with oligonucleotides being purified by high-performance liquid chromatography (HPLC) and/or denaturing polyacrylamide gel electrophoresis (PAGE) prior to their use in any calorimetry experiments. The concentrations of all nucleic acids used in calorimetric studies should be determined spectrophotometrically using experimentally derived extinction coefficients. UNIT 7.3 describes an excellent method for determining the extinction coefficient of a nucleic acid by digesting the nucleic acid (either enzymatically or 
chemically) and subsequently performing colorimetric quantification of the phosphate concentration.

\section{Differential Scanning Calorimetry}

The choice of buffer is important in performing DSC on a nucleic acid. One should not choose buffers whose $\mathrm{pK}_{\mathrm{a}}$ values exhibit large temperature dependencies (e.g., Tris.Cl; $\left.\Delta \mathrm{pK}_{\mathrm{a}} /{ }^{\circ} \mathrm{C}=-0.028\right)$. Thus, the selection of a DSC buffer should depend not only on its buffering capacity at a desired experimental $\mathrm{pH}$, but also on its $\mathrm{pK}_{\mathrm{a}}$ having a minimal temperature dependence. Examples of suitable DSC buffers include phosphoric acid $\left(\mathrm{pK}_{\mathrm{a} 2}\right.$ at $\left.25^{\circ} \mathrm{C}=7.20 ; \Delta \mathrm{pK}_{\mathrm{a} 2} /{ }^{\circ} \mathrm{C}=-0.0028\right)$, citric acid $\left(\mathrm{pK}_{\mathrm{a} 3}\right.$ at $\left.25^{\circ} \mathrm{C}=6.40 ; \Delta \mathrm{pK}_{\mathrm{a} 3} /{ }^{\circ} \mathrm{C} \approx 0\right)$, and acetic acid $\left(\mathrm{pK}_{\mathrm{a}}\right.$ at $\left.25^{\circ} \mathrm{C}=4.76 ; \Delta \mathrm{pK}_{\mathrm{a}} /{ }^{\circ} \mathrm{C}=0.002\right)$.

The choice of salt (cation) concentration is another important consideration in designing a DSC experiment. The thermal stability of a polyanionic nucleic acid molecule depends on the concentration of cation in solution. In general, the higher the cation concentration, the greater the thermal stability of the nucleic acid. Thus, one can modulate the temperature range over which the melting transition of the nucleic acid occurs by varying the salt concentration. This ability can prove useful for ensuring sufficient pre- and post-transition baseline readings for accurate analysis of the data.

Multivalent cations generally are more potent stabilizers of nucleic acid thermal stability than monovalent cations. Thus, conferring a desired thermal stability upon a nucleic acid molecule requires lower concentrations of salts containing multivalent cations-e.g., $\mathrm{MgCl}_{2}$ or $\mathrm{Co}\left(\mathrm{NH}_{3}\right)_{6} \mathrm{Cl}_{3}$ - than salts containing monovalent cations-e.g., $\mathrm{NaCl}$ or $\mathrm{KCl}$. Note that different cations of similar valence (e.g., $\mathrm{Na}^{+}$versus $\mathrm{Li}^{+}, \mathrm{Mg}^{2+}$ versus $\mathrm{Ca}^{2+}$, or $\mathrm{Co}^{3+}$ versus spermidine ${ }^{3+}$ ) can differ significantly in the extent to which they thermally stabilize a given nucleic acid structure. In spite of this variability in degree of cation-induced thermal enhancement, a good rule of thumb for oligonucleotides is to use cation concentrations in the following ranges: $50 \mathrm{mM}$ to $1 \mathrm{M}$ for monovalent cations, 1 to 15 $\mathrm{mM}$ for divalent cations, and 20 to $500 \mu \mathrm{M}$ for cations with valences of $\geq 3$. For longer DNA fragments, such as polynucleotides (particularly those having high GC contents), it may be necessary to use cation concentrations that fall below these ranges to ensure complete denaturation of the nucleic acid over the experimentally accessible temperature range, as well as to prevent cation-induced aggregation and/or precipitation of the nucleic acid.

A third important criterion in designing a DSC experiment is choosing an appropriate concentration of the nucleic acid to be studied. The dissociation heat (enthalpy) of a nucleic acid molecule depends on both its length and its base sequence. In general, the heat of dissociation decreases with decreasing fragment length. Thus, DSC experiments on short nucleic acid fragments require larger concentrations than those on longer nucleic acid fragments. The minimum concentration of a given nucleic acid fragment required for a DSC experiment will depend not only on the length and sequence of the fragment, but also on the sensitivity of the DSC instrument being employed. The Materials section of Basic Protocol 1 lists a range of nucleic acid concentrations that is suitable for most DSC instruments. Note that the thermal stabilities of short nucleic acid molecules (e.g., $\leq 20$ base pairs, base triplets, etc.) with molecularities of two or more depend on their concentrations, with increasing concentrations resulting in increased thermal stabilities. Thus, in studies on such short multimolecular nucleic acids, not only can one modulate the temperature range over which the melting transition occurs by varying the salt concentration (as noted above), but also by varying the nucleic acid concentration. 
It is critical to the success of an ITC experiment that concentrations of the solution components (i.e., buffer, salt, chelating agent) in the two nucleic acid solutions be as close to identical as possible. Such solution components can have high heats of dilution and thereby can introduce substantial error to the measurement. Similarly, one should ensure that the $\mathrm{pH}$ values of the two nucleic acid solutions are as close to identical as possible, since buffers often have substantial heats of protonation. The best way to insure that all the components in the two nucleic acid solutions are identical is to prepare a single batch of buffer solution, lyophilize an appropriate amount of each of the two nucleic acid samples to dryness, and dissolve each of the dried nucleic acid samples in the appropriate volume of buffer.

\section{DIFFERENTIAL SCANNING CALORIMETRY OF NUCLEIC ACIDS}

This protocol describes the use of DSC to study thermally induced order-disorder transitions in nucleic acids. Steps 1 to 5 describe the acquisition of a buffer-versus-buffer DSC scan, while steps 6 to 9 describe the acquisition of the corresponding nucleic acid-versus-buffer scan. Both scans are required, since the first step in data analysis mandates the background correction of the nucleic acid-versus-buffer scan by subtracting from it the corresponding buffer-versus-buffer scan. Analysis of the resulting DSC data is described below (see Commentary).

\section{Materials}

Appropriate buffer

Buffer solution of purified nucleic acid ( 0.2 to $2.0 \mathrm{mM}$ in nucleotide)

Nitrogen $\left(\mathrm{N}_{2}\right)$ gas

Differential scanning calorimetry (DSC) instrument

Vacuum source and side-arm flask (for degassing)

1. Rinse both the sample and reference chambers of the DSC instrument thoroughly with a copious volume ( 0.5 to 1.0 liter) of distilled water and dry with nitrogen gas.

2. Degas a sufficient volume (typically 3 to $4 \mathrm{~mL}$ ) of buffer to fill both the sample and reference chambers. Maintain vacuum for $\sim 10$ to $15 \mathrm{~min}$.

3. Fill both the sample and reference chambers with degassed buffer, being careful not to introduce any air bubbles.

4. Select the desired temperature limits and scan rate.

A typical scan rate for a DSC experiment on a nucleic acid is $1.0^{\circ} \mathrm{C} / \mathrm{min}$. However, if the kinetics of the order-disorder equilibrium being studied are slow, one may need to lower this temperature scan rate in order to ensure equilibrium conditions throughout the scan. Note that upon decreasing the scan rate, one must increase the nucleic acid concentration in order to maintain the same signal strength. A good rule of thumb is to double the nucleic acid concentration upon halving the scan rate.

5. Allow the instrument to equilibrate (typically 20 to $40 \mathrm{~min}$ ) and start the scan.

6. Repeat step 1.

7. Prepare a sufficient volume (typically 1.5 to $2 \mathrm{~mL}$ ) of buffer to fill the reference chamber and a sufficient volume (typically 1.5 to $2 \mathrm{~mL}$ ) of nucleic acid solution in buffer to fill the sample chamber. Degas both solutions for $\sim 10$ to $15 \mathrm{~min}$. 
8. Fill the sample chamber with the degassed nucleic acid solution and the reference chamber with the degassed buffer, taking care not to introduce any air bubbles.

9. Repeat steps 4 and 5.

$B A S I C$ PROTOCOL 2
Calorimetry of Nucleic Acids

\section{ISOTHERMAL TITRATION CALORIMETRY OF NUCLEIC ACIDS}

This protocol describes the use of ITC to study the hybridization of nucleic acid molecules at a constant temperature. Analysis of the resulting ITC data is described below (see Commentary). ITC cannot be used to study the thermodynamics of self-complementary nucleic acids. For evaluating the thermodynamics of self-complementary nucleic acids, DSC is the only calorimetric technique that can be employed.

The choice of the concentration of the titrant nucleic acid (nucleic acid $\mathbf{T}$ ) will depend on the expected stoichiometry of its interaction with the analyte nucleic acid (nucleic acid A). When this stoichiometry is 1:1 (as would be the case for two single strands hybridizing to form a duplex), a good rule of thumb is to use 25 times more titrant than analyte. This ratio will ensure that the lower and upper baselines of the resulting titration curve are well defined.

\section{Materials}

Appropriate buffer

Buffer containing purified analyte nucleic acid (nucleic acid $\mathbf{A} ; 0.1$ to $1.0 \mathrm{mM}$ in nucleotide)

Buffer containing purified titrant nucleic acid (nucleic acid $\mathbf{T}$ ), whose base sequence is complementary to that of nucleic acid $\mathbf{A}$ and whose concentration is $\geq 20$ times that of nucleic acid $\mathbf{A}$

Isothermal titration calorimetry (ITC) instrument

Vacuum source and side-arm flask (for degassing)

1. Set the ITC thermostat to the desired temperature.

If the desired temperature is at or below ambient temperature, use a refrigerated circulating water bath to facilitate the temperature regulation of the sample cell, and set the bath to a temperature $\geq 5^{\circ} \mathrm{C}$ below the desired ITC thermostat temperature. When using an external water bath, let the bath and ITC instrument equilibrate for $\sim 24 \mathrm{hr}$ prior to running an experiment.

2. Degas appropriate buffer or distilled water for $\sim 10$ to $15 \mathrm{~min}$ and use it to fill the reference cell, taking care not introduce any air bubbles.

Unless more frequent changes are warranted by corresponding changes in buffer or solvent, the solution in the reference cell need only be changed once per month.

3. Rinse the sample cell of the ITC instrument thoroughly with a copious volume $(0.5$ to 1.0 liter) of distilled water.

4. Prepare a sufficient volume of buffer solution containing nucleic acid $\mathbf{A}$ to fill the sample cell and degas for 10 to $15 \mathrm{~min}$.

5. Fill the sample cell with nucleic acid A solution, being careful not to introduce any air bubbles.

6. Allow the instrument to equilibrate (typically 20 to $40 \mathrm{~min}$ ).

7. While the instrument is equilibrating, prepare a sufficient volume of buffer solution containing nucleic acid $\mathbf{T}$ to fill the titrating syringe and degas for 10 to $15 \mathrm{~min}$. 
8. Fill the titrating syringe with nucleic acid $\mathbf{T}$ solution, being careful not to introduce any air bubbles into the syringe.

9. Keeping the syringe as upright as possible, insert the syringe into the sample cell and firmly seat into place. Align the motor-driven piston with the syringe plunger, if not done automatically by computer control.

10. Start rotating the syringe at a speed of $\sim 400 \mathrm{rpm}$ and allow the instrument to equilibrate for 20 to $40 \mathrm{~min}$.

11. After the instrument has equilibrated (i.e., the baseline is essentially unchanging), set the run parameters-including the number of injections, the injection volume, and the time between injections - and begin the titration.

To derive parameters such as $K$ and $n$ from a single ITC experiment, the resulting titration curve should be defined by $\geq 10$ points (i.e., $\geq 10$ injections).

12. Repeat steps 3 to 11, replacing the nucleic acid $\mathbf{A}$ solution in the sample cell with buffer alone.

This control experiment will yield the sequential dilution heats associated with injection of nucleic acid $\boldsymbol{T}$ into buffer, which, in turn, must be subtracted from the corresponding experimental heats resulting from the titration of nucleic acid $\boldsymbol{T}$ into nucleic acid $\boldsymbol{A}$.

13. Repeat steps 3 to 11 , replacing the nucleic acid $\mathbf{T}$ solution in the injecting syringe with a solution of buffer alone.

This control experiment will yield the sequential dilution heats, if any, associated with injection of buffer into nucleic acid $\boldsymbol{A}$. These heats often are negligible; however, in cases where they are not, they must be subtracted from the corresponding experimental heats resulting from the titration of nucleic acid $\boldsymbol{T}$ into nucleic acid $\boldsymbol{A}$.

\section{COMMENTARY}

\section{Background Information}

In recent years, it has become apparent that thermodynamic as well as structural information is essential for understanding the nature of the relationships between the structures, energetics, and biological functions of nucleic acids. Recognition of this need, coupled with the commercial availability of sensitive calorimetric instruments, has led to a profound increase in the number of studies exploring the thermodynamics of nucleic acids (Sturtevant, 1987; Breslauer et al., 1992; Plum and Breslauer, 1995).

Differential scanning and isothermal titration calorimetry are the two major calorimetric techniques that are currently employed to characterize the thermodynamics of nucleic acids. The purpose of this unit is to provide specific protocols for applying these two calorimetric techniques to the study of nucleic acid hybridization. Consequently, no attempt is made to review the theory of calorimetry or to present a description of instrument features. For such information, refer to the instruction manuals for the calorimeters, previously published reviews
(Sturtevant, 1987; Freire et al., 1990; Breslauer et al., 1992; Plum and Breslauer, 1995), as well as the original research articles referenced in these manuals and reviews.

\section{Differential scanning calorimetry}

Differential scanning calorimetry (DSC) is used to study thermally induced order-disorder transitions in nucleic acids. A DSC instrument measures as a function of temperature $(T)$ the excess heat capacity $\left(C_{\mathrm{p}}^{\mathrm{ex}}\right)$ of a nucleic acid solution relative to the same amount of buffer solution. As detailed below, a single DSC profile provides a wealth of both thermodynamic and extrathermodynamic information, much of which cannot be obtained by any other technique.

Integration of the experimental curve of $C_{\mathrm{p}}^{\mathrm{ex}}$ versus $T$ (see example in Fig. 7.4.1) yields the transition enthalpy $(\Delta H)$, since $\Delta H=$ $\int C_{\mathrm{p}} \mathrm{d} T$. Note that this calorimetrically determined transition enthalpy $\left(\Delta H_{\text {cal }}\right)$ is model independent and therefore does not depend on the state of the transition (two-state, multistate). This characteristic distinguishes $\Delta H_{\mathrm{cal}}$ from
Biophysical Analysis of Nucleic Acids 
model-dependent van't Hoff transition enthalpies $\left(\Delta H_{\mathrm{vH}}\right)$, which are derived from the temperature dependence of equilibrium properties, and are typically predicated on the assumption (model) that the transition proceeds in an allor-none (two-state) fashion, with no thermodynamic contributions from intermediate states. Citing disparities that often arise between $\Delta H_{\mathrm{vH}}$ and $\Delta H_{\text {cal }}$, Sturtevant and co-workers (Naghibi et al., 1995) have stressed the importance of using model-independent calorimetric measurements rather than less-reliable, model-dependent van't Hoff analyses to characterize the thermodynamics of biological macromolecules.

The heat capacity change $\left(\Delta C_{\mathrm{p}}\right)$ for the transition can be derived from the difference between pre- and posttransition baselines in a DSC measurement (Edsall and Gutfreund, 1983). This difference is often negligible for nucleic acids (i.e., $\Delta C_{\mathrm{p}} \approx 0$ ), as is the case for the DSC profile shown in Figure 7.4.1. In cases where the experimental curve of $C_{\mathrm{p}}^{\mathrm{ex}}$ versus $T$ yields an insufficient amount of pre- or posttransition baseline for accurate integration and/or $\Delta C_{\mathrm{p}}$ analysis, the salt and/or nucleic acid concentration can be modified (see Strategic Planning) to shift the transition to a higher or lower temperature range, as needed.

The experimental curve of $C_{\mathrm{p}}^{\mathrm{ex}}$ versus $T$ can be converted to $C_{\mathrm{p}}^{\text {ex }} / T$ versus $T$ by dividing the raw $C_{\mathrm{p}}^{\mathrm{ex}}$ data by $T$ and then replotting the resulting values as a function of $T$ (Marky and

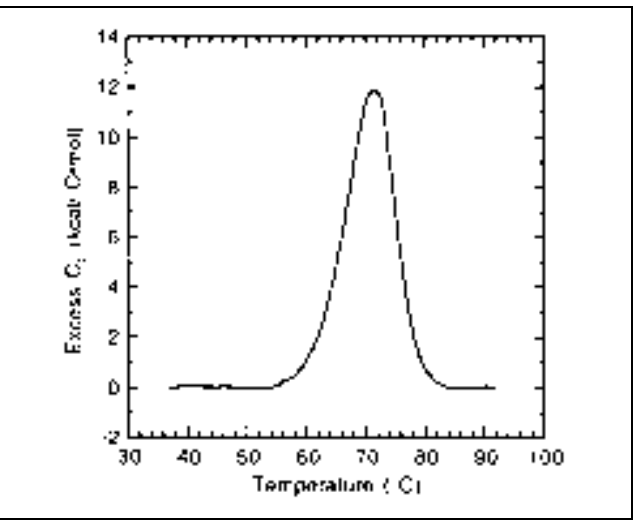

Figure 7.4.1 DSC profile for the thermal denaturation of the $d$ (CCTCTCCGGCTCTTC). d(GAAGAGCCGGAGAGG) duplex. This DSC measurement was conducted on a model 5100 Nano Differential Scanning Calorimeter (Calorimetry Sciences) using a temperature scanning rate of $60^{\circ} \mathrm{C} / \mathrm{hr}$. The DNA concentration was $50 \mu \mathrm{M}$ in duplex and the solution conditions were $10 \mathrm{mM}$ sodium cacodylate ( $\mathrm{pH} 7.0), 100$ $\mathrm{mM} \mathrm{NaCl}, 10 \mathrm{mM} \mathrm{MgCl}_{2}$, and $0.1 \mathrm{mM}$ EDTA.
Breslauer, 1987). Integration of this curve yields the transition entropy $(\Delta S)$, since $\Delta S=$ $\int\left(C_{\mathrm{p}} / T\right) \mathrm{d} T$. Thus, a single DSC curve can yield $\Delta H, \Delta S$, and $\Delta C_{\mathrm{p}}$. Once these data are known, the corresponding transition free energy $(\Delta G)$ can be determined at any temperature $(T)$ using the following general thermodynamic relationship (Edsall and Gutfreund, 1983; Breslauer et al., 1992): $\Delta G=\Delta H-T \Delta S$. Note that although $\Delta S$ and $\Delta G$ can be extracted from DSC data, these data are less reliable than the $\Delta H$ and $\Delta C_{\mathrm{p}}$ values obtained directly, due to the coupling and propagation of errors (Krug et al., 1976).

$\Delta H_{\mathrm{vH}}$ can be determined by analysis of the shape (either the full or half width at half height) of an experimental curve of $C_{\mathrm{p}}^{\mathrm{ex}}$ versus $T$ using either of the following two relationships (Gralla and Crothers, 1973; Breslauer, 1995):

For full width at half height:

$$
\Delta H_{\mathrm{vH}}=\frac{\mathrm{B}}{\frac{1}{T_{1}}-\frac{1}{T_{2}}}
$$

\section{Equation 7.4.1}

For upper half width at half height:

$$
\Delta H_{\mathrm{vH}}=\frac{B^{\prime}}{\frac{1}{T_{\max }}-\frac{1}{T_{2}}}
$$

\section{Equation 7.4.2}

where $T_{\max }$ is the temperature at the maximum of the experimental curve of $C_{\mathrm{p}}^{\mathrm{ex}}$ versus $T$, and $T_{1}$ and $T_{2}$ correspond to the lower and upper temperatures, respectively, at which $C_{\mathrm{p}}^{\mathrm{ex}}$ is equal to one half of the maximum value. $B$ and $B^{\prime}$ are constants that depend on the molecularity of the melting process under investigation. For a bimolecular process, such as the thermally induced denaturation of a DNA duplex into two single strands (as depicted by the DSC curve shown in Fig. 7.4.1), $B$ and $B^{\prime}$ are equal to 10.14 and $4.38 \mathrm{cal} / \mathrm{mol} \cdot \mathrm{K}$, respectively.

A comparison of $\Delta H_{\mathrm{vH}}$ and $\Delta H_{\mathrm{cal}}$ allows one to evaluate the state of the transition (Marky and Breslauer, 1987). Specifically, if $\Delta H_{\mathrm{vH}}=$ $\Delta H_{\text {cal }}$, then the transition proceeds in a twostate, all-or-none fashion. Under such conditions, meaningful thermodynamic data can be obtained from van't Hoff analyses of equilibrium data. However, if $\Delta H_{\mathrm{vH}}<\Delta H_{\text {cal }}$, then the transition involves intermediate states, thereby precluding the use of the two-state van't Hoff model. If $\Delta H_{\mathrm{vH}}>\Delta H_{\mathrm{cal}}$, then intermolecular cooperation (e.g., aggregation) is indicated. A comparison of $\Delta H_{\mathrm{vH}}$ and $\Delta H_{\mathrm{cal}}$ also provides insight into the cooperative nature of the tran- 
sition. Specifically, the ratio $\Delta H_{\mathrm{vH}} / \Delta H_{\text {cal }}$ provides a measure of the fraction of the structure that melts as a single thermodynamic entity (i.e., the size of the cooperative unit).

\section{Isothermal titration calorimetry}

Isothermal titration calorimetry (ITC) is used to study the hybridization of nucleic acid molecules at a constant temperature (Wiseman et al., 1989; Freire et al., 1990; Breslauer et al., 1992; Wilson et al., 1994; Plum and Breslauer, 1995; Plum et al., 1995). In a typical ITC experiment, small aliquots of a titrant nucleic acid solution are injected into an analyte nucleic acid solution (with the analyte nucleic acid being complementary to the titrant nucleic acid) and the heat of hybridization is measured directly. Each injection results in a heat burst curve, as depicted in Figure 7.4.2A. The heat

$$
\frac{\partial(\Delta Q)}{\partial\left(\left[\mathrm{T}_{\mathrm{tot}}\right)\right.}=\Delta H \times V\left\{\frac{1}{2}+\frac{1-\frac{1+\left(\frac{1}{K\left[\mathrm{~A}_{\mathrm{tot}}\right]}\right)}{2}-\frac{\left[T_{\mathrm{tot}}\right]}{2\left[\mathrm{~A}_{\mathrm{tot}}\right]}}{\sqrt{\left(\frac{\left[T_{\mathrm{tot}}\right]}{\left[\mathrm{A}_{\mathrm{tot}}\right]}\right)^{2}-2 \frac{\left[T_{\mathrm{tot}}\right]}{\left[\mathrm{A}_{\mathrm{tot}}\right]}\left(1-\frac{1}{K\left[\mathrm{~A}_{\mathrm{tot}}\right]}\right)+\left(1-\frac{1}{K\left[\mathrm{~A}_{\mathrm{tot}}\right]}\right)^{2}}}\right\}
$$

\section{Equation 7.4.3}

Once the value of $K$ has been determined in this manner, the free energy $(\Delta G)$ of association can be derived from the relationship $\Delta G=$ $-R T \ln K$. The resulting value of $\Delta G$ then can be used in conjunction with $\Delta H$ to derive the entropy $(\Delta S)$ of association using the following standard relationship: $\Delta S=(\Delta H-\Delta G) / T$.

Thus, as noted above for DSC, a single ITC experiment also yields a wealth of thermodynamic information about the association reaction. The heat capacity change, $\Delta C_{\mathrm{p}}$, for the association reaction can be determined by repeating the ITC experiment at two different temperatures using the following thermodynamic relationship:

$$
\Delta C_{\mathrm{p}}=\frac{\partial \Delta H}{\partial T}=\frac{\Delta H_{\mathrm{T}_{2}}-\Delta H_{\mathrm{T}_{1}}}{T_{2}-T_{1}}
$$

\section{Equation 7.4.4}

where $T_{1}$ and $T_{2}$ are the two different temperatures at which the ITC experiments were conducted. Note that $K$ can be accurately derived from an ITC titration curve $(\Delta Q$ versus $\left.\left[\mathrm{T}_{\text {tot }}\right] /\left[\mathrm{A}_{\text {tot }}\right]\right)$ only if $K \leq 10^{8} \mathrm{M}^{-1}$. When $K>10^{8}$ $\mathrm{M}^{-1}$ (as is often observed when two complementary nucleic acid strands hybridize to form a duplex), the extreme sharpness of the ITC titration curve precludes accurately fitting for
( $\Delta Q$; expressed in kcal or $\mathrm{kJ}$ per mole of injected titrant) evolved from each injection can be determined by integration of the corresponding heat burst curves and subsequently plotted as a function of the molar ratio of the two interacting nucleic acids $\left(\left[\mathrm{T}_{\mathrm{tot}}\right] /\left[\mathrm{A}_{\mathrm{tot}}\right]\right.$; see Fig. 7.4.2B). The resulting titration curve can be analyzed by nonlinear least squares fitting to yield the stoichiometry of the association reaction $(n)$, the enthalpy of association $(\Delta H)$, and the equilibrium association constant $(K)$ (Wiseman et al., 1989; Freire et al., 1990). For a reaction with a $1: 1$ stoichiometry $(n=1)$, as is the case when two complementary nucleic acid strands hybridize to form a duplex, a plot of $\Delta Q$ versus $\left[\mathrm{T}_{\text {tot }}\right] /\left[\mathrm{A}_{\text {tot }}\right]$ can be fit to the following equation to yield $\Delta H$ and $K$ (Wiseman et al., 1989), where $V$ is the volume of the sample cell.

$K$. In such cases, it is better to use DSC to evaluate the hybridization thermodynamics.

Caution should be exercised when comparing the thermodynamic parameters derived from ITC data with those derived from DSC data, since the single-stranded states of the nucleic acids exist at lower thermal energy $(\mathrm{k} T)$ in ITC experiments than they do in DSC experiments. The Breslauer group (Vesnaver and Breslauer, 1991) has shown that, for DNA duplex formation, thermodynamic data derived from ITC and DSC experiments can be quite different due to differences in the low- and high-temperature states of the DNA single strands. Recent studies by Sarai and co-workers (Kamiya et al., 1996) have revealed that the conformational states of single-stranded DNA also play a role in the thermodynamics of DNA triplex formation.

\section{Critical Parameters}

As noted above (see Strategic Planning), two of the most critical parameters for both the DSC and ITC protocols described in this unit are the purity of the nucleic acids being studied and the accuracy with which the concentrations of the experimental nucleic acid solutions have been determined. All nucleic acids should be devoid of protein contaminants, with oligonucleotides being purified by HPLC and/or PAGE
Biophysical Analysis of Nucleic Acids 

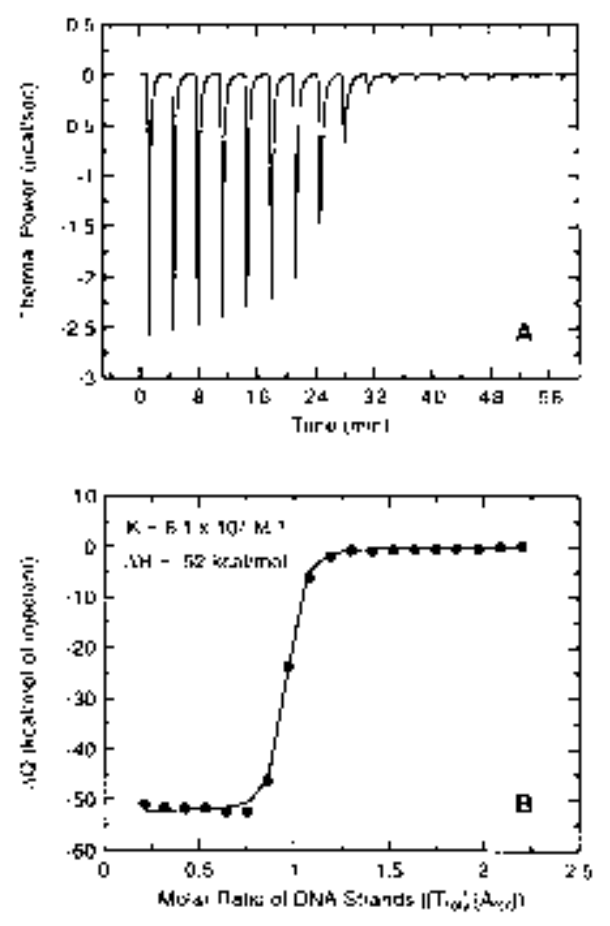

Figure 7.4.2 (A) ITC profile for the hybridization of d(CGTGTCCAGC) and d(GCTGGACACG) at $20^{\circ} \mathrm{C}$. This ITC measurement was conducted on a MicroCal model MCS Titration Calorimeter (MicroCal). Five-microliter aliquots of a d(CGTGTCCAGC) solution $(233.5 \mu \mathrm{M}$ in strand) were sequentially injected from a 100$\mu \mathrm{L}$ rotating syringe (400 rpm) into $1.31 \mathrm{~mL}$ of a d(GCTGGACACG) solution ( $8.4 \mu \mathrm{M}$ in strand). The duration of each injection was $4.93 \mathrm{sec}$ and the delay between injections was $200 \mathrm{sec}$. The solution conditions were $10 \mathrm{mM}$ sodium cacodylate ( $\mathrm{pH} 7.0), 10 \mathrm{mM} \mathrm{KCl}, 10 \mathrm{mM} \mathrm{MgCl}$, and $5 \mathrm{mM} \mathrm{CaCl}_{2}$. (B) Integrated areas of each heat burst curve in (A) plotted as a function of the molar ratio of $d(C G T G T C C A G C)$ to d(GCTGGACACG). The solid line reflects the nonlinear least squares fit of the data to Equation 7.4.3, where $K=6.1 \times 10^{7} \mathrm{M}^{-1}$ and $\Delta H=$ $-52 \mathrm{kcal} / \mathrm{mole}$.

prior to their use in any calorimetry experiments. The concentrations of all nucleic acids used in calorimetric studies should be determined spectrophotometrically using experimentally derived extinction coefficients.

For DSC experiments, choosing the correct buffer is of critical importance. One should not choose buffers whose $\mathrm{pK}_{\mathrm{a}}$ values exhibit large temperature dependencies (e.g., Tris.Cl, $\left.\Delta \mathrm{pK}_{\mathrm{a}}{ }^{\circ} \mathrm{C}=-0.028\right)$. Thus, selecting a DSC buffer should depend not only on its buffering capacity at a desired experimental $\mathrm{pH}$, but also on its $\mathrm{pK}_{\mathrm{a}}$ having minimal temperature depend- ence. Examples of suitable DSC buffers include phosphoric acid $\left(\mathrm{pK}_{\mathrm{a} 2}\right.$ at $25^{\circ} \mathrm{C}=7.20$; $\left.\Delta \mathrm{pK}_{\mathrm{a} 2}{ }^{\circ} \mathrm{C}=-0.0028\right)$, citric acid $\left(\mathrm{pK}_{\mathrm{a} 3}\right.$ at $25^{\circ} \mathrm{C}$ $\left.=6.40 ; \Delta \mathrm{pK}_{\mathrm{a} 3} /{ }^{\circ} \mathrm{C} \approx 0\right)$, and acetic acid $\left(\mathrm{pK}_{\mathrm{a}}\right.$ at $25^{\circ} \mathrm{C}=4.76 ; \Delta \mathrm{pK}_{\mathrm{a}}{ }^{\circ} \mathrm{C}=0.002$ ). Also of importance in a DSC experiment is selection of a temperature scan rate that ensures equilibrium conditions throughout the scan.

For ITC experiments, it is of critical importance that the concentrations of the solution components (i.e., buffer, salt, chelating agent) in both the titrant and analyte nucleic acid solutions be as close to identical as possible. Such solution components can have high heats of dilution and thereby can introduce substantial error to the measurement. Similarly, one also should ensure that the $\mathrm{pH}$ values of the two nucleic acid solutions are as close to identical as possible, since buffers often have substantial heats of protonation.

\section{Anticipated Results}

DSC enables one to measure the heat capacity $\left(C_{\mathrm{p}}\right)$ of a nucleic acid in solution as a function of temperature. Integration of the resulting $C_{\mathrm{p}}$-versus- $T$ curve yields the enthalpy change associated with the thermal denaturation of the nucleic acid. Comparison of this enthalpy value with the corresponding van't Hoff enthalpy value, which can be obtained by analyzing the shape of the $C_{\mathrm{p}}$-versus- $T$ curve, allows one to evaluate both the state (e.g., two-state versus multistate) and the cooperativity of the transition. Dividing the experimentally observed $C_{\mathrm{p}}$ by $T$ and plotting the resultant data as a function of $T$ produces a $C_{\mathrm{p}} / T$-versus- $T$ curve, the integration of which yields the transition entropy $(\Delta S)$. Once $\Delta S$ and $\Delta H$ are known, the transition free energy $(\Delta G)$ can be calculated.

ITC allows one to study the hybridization of two non-self-complimentary nucleic acids at a constant temperature. Specifically, one can measure the heat evolved from sequential injections of a nucleic acid titrant solution into a solution containing the complimentary nucleic acid (the analyte nucleic acid) to the titrant. Using nonlinear least squares analysis, one can fit a plot of the sequential injection heats as a function of the molar ratio of the two nucleic acids to yield the stoichiometry $(n)$, enthalpy $(\Delta H)$, and equilibrium association constant $(K)$ for the hybridization reaction. Once $\Delta H$ and $K$ are known, the free energy $(\Delta G)$ and entropy $(\Delta S)$ of the hybridization reaction can be calculated. Running the ITC experiment at two different temperatures allows one to determine the 
heat capacity change $\left(\Delta C_{\mathrm{p}}\right)$ associated with the hybridization reaction.

\section{Time Considerations}

In general, both calorimetric protocols described in this unit are fairly rapid, often requiring no more than 1 day to complete.

\section{Literature Cited}

Breslauer, K.J. 1995. Extracting thermodynamic data from equilibrium melting curves for oligonucleotide order-disorder transitions. Methods Enzymol. 259:221-242.

Breslauer, K.J., Freire, E., and Straume, M. 1992. Calorimetry: A tool for DNA and ligand-DNA studies. Methods Enzymol. 211:533-567.

Edsall, J.T. and Gutfreund, H. 1983. Calorimetry, heat capacity, and phase transitions. In Biothermodynamics: The Study of Biochemical Processes at Equilibrium, pp. 210-227. John Wiley \& Sons, New York.

Freire, E., Mayorga, O.L., and Straume, M. 1990. Isothermal titration calorimetry. Anal. Chem. 62:950A-959A.

Gralla, J. and Crothers, D.M. 1973. Free energy of imperfect nucleic acid helices III. Small internal loops resulting from mismatches. J. Mol. Biol. 78:301-319.

Kamiya, M., Torigoe, H., Shindo, H., and Sarai, A. 1996. Temperature dependence and sequence specificity of DNA triplex formation: An analysis using isothermal titration calorimetry. J. Am. Chem. Soc. 118:4532-4538.

Krug, R.R., Hunter, W.G., and Grieger, R.A. 1976. Enthalpy-entropy compensation. 1. Some fundamental statistical problems associated with the analysis of van't Hoff and Arrhenius data. $J$. Phys. Chem. 80:2335-2341.
Marky, L.A. and Breslauer, K.J. 1987. Calculating thermodynamic data for transitions of any molecularity from equilibrium melting curves. Biopolymers 26:1601-1620.

Naghibi, H., Tamura, A., and Sturtevant, J.M. 1995. Significant discrepencies between van't Hoff and calorimetric enthalpies. Proc. Natl. Acad. Sci. U.S.A. 92:5597-5599.

Plum, G.E. and Breslauer, K.J. 1995. Calorimetry of proteins and nucleic acids. Curr. Opin. Struct. Biol. 5:682-690.

Plum, G.E., Pilch, D.S., Singleton, S.F., and Breslauer, K.J. 1995. Nucleic acid hybridization: Triplex stability and energetics. Annu. Rev. Biophys. Biomol. Struct. 24:319-350.

Sturtevant, J.M. 1987. Biochemical applications of differential scanning calorimetry. Аnnu. Rev. Phys. Chem. 38:463-488.

Vesnaver, G. and Breslauer, K.J. 1991. The contribution of DNA single-stranded order to the thermodynamics of duplex formation. Proc. Natl. Acad. Sci. U.S.A. 88:3569-3573.

Wilson, W.D., Hopkins, H.P., Mizan, S., Hamilton, D.D., and Zon, G. 1994. Thermodynamics of DNA triplex formation in oligomers with and without cytosine bases: Influence of buffer species, $\mathrm{pH}$, and sequence. J. Am. Chem. Soc. 116:3607-3608.

Wiseman, T., Williston, S., Brandts, J.F., and Lin, L.-N. 1989. Rapid measurement of binding constants and heats of binding using a new titration calorimeter. Anal. Biochem. 179:131-137.

Contributed by Daniel S. Pilch

University of Medicine and Dentistry of New Jersey

Robert Wood Johnson Medical School Piscataway, New Jersey
Biophysical Analysis of Nucleic Acids 